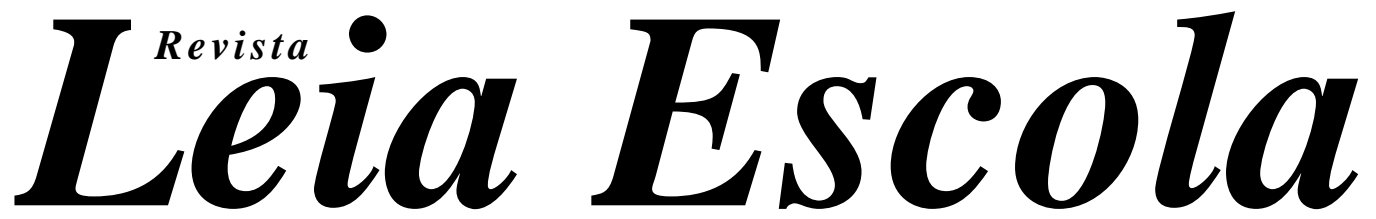

Programa de Pós-Graduação em Linguagem e Ensino 
UNIVERSIDADE FEDERAL DE CAMPINA GRANDE

Reitor Vicemário Simões

Vice-reitor Camilo Allyson Simões de Farias

Diretora do Centro de Humanidades Fernanda de Lourdes Almeida Leal

UNIDADE ACADÊMICA DE LETRAS

Coordenadora Administrativa Márcia Candeia Rodrigues

Coordenadores de Graduação

Licenciatura em Letras Espanhol

Licenciatura em Letras Francês

Licenciatura em Letras Inglês

Licenciatura em Letras Libras

Licenciatura em Letras Português

Coordenadora do Programa de Pós-Graduação em

Linguagem e Ensino

Coordenadora de Pesquisa e Extensão

Isis Milreu

Carmen Verônica A. R. Nóbrega

Cleydstone Chaves dos Santos

Aline Risseli Florindo Silva

Edmilson Luiz Rafael

Manassés Morais Xavier

Denise Lino de Araújo

Neide de Fátima Cesar da Cruz

Editora da Universidade Federal de Campina Grande Diretor Administrativo Revista Leia Escola Editora Chefe

Comissão Editorial Quadriênio 2018-2021

José Hélder Pinheiro Alves

ISSN 2358-5870

Márcia Tavares

Denise Lino de Araújo

Maria Augusta G. de Macedo Reinaldo

Naelza de Araújo Wanderley

Corpo Editorial Aldinida Medeiros (UEPB)

André Pinheiro (UFPI)

Angela Paiva Dionisio (UFPE)

Amarino Queiroz (UFRN)

Ana Cristina Marinho Lúcio (UFPB)

Ana Virgínia Lima (UFRN)

Bruno Alves Pereira (UEPB)

Darcília Simões (UERJ)

Daniela Segabinazi (UFPB)

Derivaldo dos Santos (UFRN)

Edenize Ponzo Peres (UFES)

Edmilson Luiz Rafael (UFCG)

Eliana Kefalas Oliveira (UFAL)

Fabiana Oliveira (UFAL)

Fabiele Stockmans de Nardi (UFPE)

Fabiano Tadeu Grazioli (URI/FAE)

Fábio Marques de Souza (UEPB)

Fátima Aparecida Teves Cabral Bruno (USP)

Fernanda Aquino Sylvestre (UFU)

Givaldo Melo de Santana (UFSE)

Josilene Pinheiro Mariz (UFCG)

José Hélder Pinheiro Alves (UFCG)

Karina Chianca (UFPB)

Lílian de Oliveira Rodrigues (UERN)

Luiz Francisco Dias (UFMG)

Maria Amélia Dalvi (UFES)

Marco Antônio Margarido Costa (UFCG)

Maria Angélica de Oliveira (UFCG)

Maria Auxiliadora Bezerra (UFCG)

Maria Hozanete Alves de Lima (UFRN)

Maria Marta dos Santos Silva Nóbrega (UFCG)

Marie Hélène Torres (UFSC)

Renata Junqueira de Souza (UNESP)

Roberto Carlos de Assis (UFPB)

Sílvia Pilegi Rodrigues (UFMT)

Simone Dália de Gusmão Aranha (UEPB)

Sinara de Oliveira Branco (UFCG)

Valdenides Cabral Dias (UFRN)

Capa: Helena Denardi

Os trabalhos publicados são de responsabilidade exclusiva dos seus autores $\quad$ ISSN 2358-5870

Leia Escola - Revista do Programa de Pós-Graduação em Linguagem e Ensino da UFCG.

v. 20, n. 1, 2020. Campina Grande: EDUFCG, 2020.

1. Linguística

2. Linguística Aplicada

3. Literatura

4. Ensino 
UNIVERSIDADE FEDERAL DE CAMPINA GRANDE CENTRO DE HUMANIDADES

UNIDADE ACADÊMICA DE LETRAS
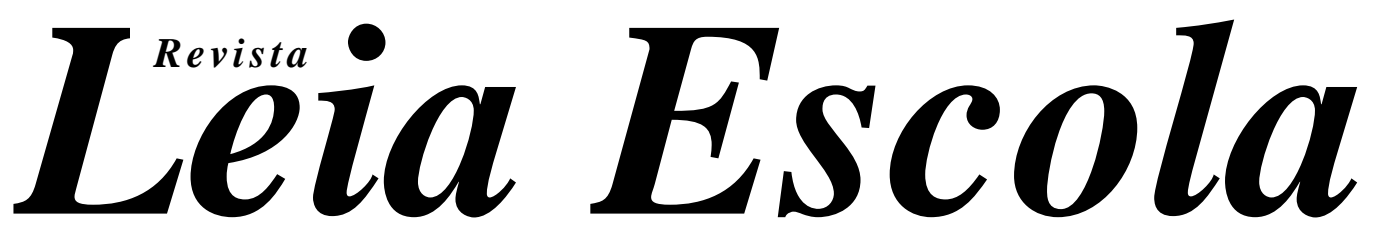

Programa de Pós-Graduação em Linguagem e Ensino

\title{
JOGOS, LETRAMENTOS E ÉTICA
}

\author{
Organizadoras \\ Angela Paiva Dionisio \\ Maria de Lourdes Dionisio \\ Regina Lúcia Peret Dell'Isola Denardi \\ Tatyana Mabel Nobre Barbosa
}

EDUFCG

Campina Grande

2020 


\section{SUMÁRIO}

APRESENTAÇÃO

\section{DOSSIÊ TEMÁTICO}

ÁRIDA: O DESPERTAR DO SERTÃO E A EXPRESSÃO DA CULTURA NORDESTINA ATRAVÉS DOS ELEMENTOS CONSTITUTIVOS DE UM JOGO DIGITAL

Bruna Maele Girão Pinheiro, Regina Cláudia Pinheiro e Júlio Araújo.

ANÁLISE LINGUÍSTICA MEDIADA POR JOGOS

Kiahra Antonella Coelho Pereira, Wagner Rodrigues Silva e Jose Robson Mariano Alves.

ANÁLISE DO JOGO "LIFE IS STRANGE" E SUAS POTENCIALIDADES DE INTERAÇÃO COM O SUJEITO-LEITOR/ JOGADOR

Isabela Vieira Lima, Helena Maria Ferreira

UMA ANÁLISE DA FERRAMENTA ALICE NO ENSINO DA LÓGICA DE PROGRAMAÇÃO

Márcia Antônia Dias Catunda, Mayumi Passos Lopes e José Aires de Castro Filho.

POSSIBILIDADES PEDAGÓGICAS DE FORMAÇÃO DO LEITOR CRÍTICO DE TEXTOS LITERÁRIOS A PARTIR DE UM JOGO DIGITAL PARA ALUNOS DO ENSINO MÉDIO.

Rodrigo Alves dos Santos

O JOGO DE TABULEIRO NO CONTEXTO DA EDUCAÇÃO LIBERTADORA E DO ENSINO POR COMPETÊNCIA

Laura Dourado Loula Regis.

JOGOS DIGITAIS DE ALFABETIZAÇÃO: UM PANORAMA PEDAGÓGICO DE ALGUMAS PRODUÇÕES ACADÊMICAS BRASILEIRAS

Chris Royes Schardosim, Thiago Ribeiro Alves

O CONHECIMENTO HISTÓRICO ENTRE MAPAS E JOGOS: O ENSINO DE GRÉCIA ANTIGA A PARTIR DO JOGO DE TABULEIRO

Carolina Ferreira de Figueiredo, Lucas Werlang Girardi.

\section{ARTIGOS}

O ENSINO DE PRONÚNCIA NAS AULAS DE ESPANHOL NO COLÉGIO PEDRO II CRENÇAS E PRÁTICAS DOCENTES

José Ricardo Dordron de Pinho.

A DOCÊNCIA NO ESTÁGIO PÓS-DOUTORAL COMO PROCESSO DE FORMAÇÃO PROFISSIONAL: RELATO DE EXPERIÊNCIA

Sergio Vale da Paixão, Renilson José Menegassi. 
DESENVOLVIMENTO DA CONSCIÊNCIA METATEXTUAL SOBRE A ESTRUTURA TÓPICA DO PARÁGRAFO

Marcus Vinicius Brotto de Almeida.

O USO DE TECNOLOGIAS DIGITAIS NO PLANEJAMENTO DE PROFESSORES DE INGLÊS EM PERÍODO DE ESTÁGIO SUPERVISIONADO

Michael Gouveia S.Júnior, Francisco Gabriel Cordeiro Silva, Marco Antônio Margarido Costa

ENSINO DE LÍNGUA ESTRANGEIRA NA CONTEMPORANEIDADE: NOVAS IMPLICAÇÕES NO ÂMBITO DA PRÁTICA PEDAGÓGICA

Aline Pereira Sousa, Aluíza Alves de Araújo.

LETRAMENTOS DIGITAIS EM PLANOS DE AULA DO PORTAL NOVA ESCOLA

Isabel Cristina da Silva Carneiro, Williany Miranda da Silva

ADIVINHAS EM SALA DE AULA: O BRINCAR COMO ESTÍMULO À FORMAÇÃO DE LEITORES

Leideane Faustino, José Hélder Pinheiro Alves

\section{RESENHA}

LEXICOLOGIA E SEMÂNTICA LEXICAL

Herbertt Neves

\section{ENTREVISTAS}

OS DESAFIOS DO ENSINO REMOTO NA EDUCAÇÃO BÁSICA com Denise Lino Patrícia Silva Rosas de Araújo, Paulo Ricardo Ferreira Pereira

JOGOS EM CENA com Magda Soares, Maria de Lourdes Dionísio, Renata Junqueira de Souza, Hélder Pinheiro, Regina Lúcia Peret Dell'Isola, Wagner Rodrigues Silva, Daniela Tavares Gontijo Fabiane Verardi Burlamarque, Regina Cláudia Pinheiro 